\title{
Quantitative Analysis of Retinal Microvascular Perfusion and Novel Biomarkers of the Treatment Response in Diabetic Macular Edema
}

\author{
Young Gun Park ${ }^{1}$ and Young-Hoon Park $\mathbb{D}^{2}$ \\ ${ }^{1}$ Department of Ophthalmology and Visual Science, Seoul St. Mary's Hospital, College of Medicine, The Catholic University of Korea, \\ Seoul, Republic of Korea \\ ${ }^{2}$ Catholic Institute for Visual Science, College of Medicine, The Catholic University of Korea, Seoul, Republic of Korea \\ Correspondence should be addressed to Young-Hoon Park; parkyh@catholic.ac.kr
}

Received 5 August 2020; Revised 27 October 2020; Accepted 2 November 2020; Published 17 November 2020

Academic Editor: Irini Chatziralli

Copyright ( 2020 Young Gun Park and Young-Hoon Park. This is an open access article distributed under the Creative Commons Attribution License, which permits unrestricted use, distribution, and reproduction in any medium, provided the original work is properly cited.

\begin{abstract}
Purpose. We aimed to assess the changes of retinal microvascular parameters using optical coherence tomography angiography (OCTA) between diabetes macular edema (DME) and controls. We assessed the changes between the baseline microvascular parameters and final treatment response in patients with DME, initially treated with intravitreal dexamethasone (DEX) implant followed by antivascular endothelial growth factor (VEGF) injections on an as-needed basis. Methods. This retrospective study included 90 DME patients and 24 healthy control subjects. All subjects had their best-corrected visual acuity (BCVA) and central macular thickness (CMT) measured at baseline and after 12 months. Vessel density (VD) in the superficial capillary plexus (SCP) and deep capillary plexus (DCP) and the deep/superficial flow ratio at baseline were analyzed. A subgroup analysis was used to compare the treatment response. A poor-response group was defined by five or more retreatments at 12 months. Results. BCVA and CMT showed a significant improvement at 12 months (all $p<0.001$ ). The VD in the whole and parafoveal areas of the DCP was significantly reduced in DME patients compared to that in controls (all $p<0.05)$. The DCP/SCP flow ratio was also significantly reduced in the DME group $(1.08 \pm 0.03$ vs. $1.05 \pm 0.02, p=0.001)$. In the subgroup analysis, the VD in the foveal and whole DCP areas was significantly lower in the poor-response group than that in the good-response group ( $p=0.043$ and $p=0.048$, respectively). The DCP/SCP flow ratio was also significantly lower in the poor-response group $(p=0.011)$. Conclusion. DME correlated with significant retinal microvascular impairment in the DCP. A decreased DCP/SCP flow ratio was observed in patients with DME that exhibited a poor treatment response. Retinal microvascular parameters could predict the treatment response in DME and help optimize clinical outcomes.
\end{abstract}

\section{Introduction}

Diabetic macular edema (DME), macular thickening due to diabetic retinopathy (DR), can present at any stage of this disease. It is caused by a blood-retinal barrier defect that leads to vascular leakage and fluid accumulation [1]. This process is the outcome of the expression of inflammatory factors, including vascular endothelial growth factor (VEGF), intercellular adhesion molecule-1, interleukin-6, and monocyte chemotactic protein-1, and leukostasis $[2,3]$. Because DME can cause vision loss in severe cases, it is becoming an important public health issue [4].

Many different treatment options for DME have been developed, including anti-VEGF agents and corticosteroids $[5,6]$. Intravitreal injection of anti-VEGF agents is a standard treatment for DME approved by the United States Food and Drug Administration [5]. However, this treatment poses a heavy financial burden on patients because of the numerous 
required injections during the year. On the other hand, intravitreal dexamethasone (DEX) implants $(0.7 \mathrm{mg}$ ) (Ozurdex, Allergan, Inc., Irvine, CA, USA) consist of a biodegradable copolymer that slowly releases steroids over a period of approximately 4-6 months [7].

Recently, with the increased use of optical coherence tomography (OCT) and OCT angiography (OCTA), several studies have reported various imaging biomarkers and their association with the treatment response in $\operatorname{DME}[8,9]$. OCTA allows the acquisition of images of the retinal microvasculature with good reproducibility and repeatability in a safe, rapid, and noninvasive manner. However, OCTA studies on the foveal microvascular impairment in DME are limited compared with those using OCT. Previous OCTA studies have only reported on the changes in the foveal avascular zone and the impairment of foveal microcirculation in eyes with DR $[10,11]$.

Based on these results, we aimed to evaluate the changes in retinal microvascular parameters between DME patients and healthy controls. We assessed the differences between the baseline microvascular parameters and final treatment response in DME patients, initially treated with intravitreal DEX implant followed by anti-VEGF injections on an asneeded basis.

\section{Materials and Methods}

2.1. Ethical Considerations. All procedures were conducted in accordance with the Declaration of Helsinki and its later amendments. The study was approved by the Ethics Committee of Seoul St. Mary's Hospital and the Catholic University of Korea. The requirement for informed patient consent was waived due to the retrospective design of the study.

2.2. Study Design and Subjects. This study was a retrospective review of consecutive patients who attended the Department of Ophthalmology of Seoul St. Mary's Hospital between January 2017 and January 2019. The study included patients with a diagnosis of diabetes mellitus and DME, who received a DEX implant injection and were followed up for at least 12 months. The healthy control group included healthy patients with no posterior segment abnormalities or systemic comorbidities who attended medical checkups.

The exclusion criteria for patients with DME were as follows: (1) any other ocular disease that may affect ocular circulation (e.g., glaucoma, age-related macular degeneration, and refractive error $>5$ diopters); (2) intraocular pressure > $25 \mathrm{mmHg}$; (3) severe media opacity (e.g., lens opacity due to cataract or thick asteroid hyalosis); (4) macular edema due to any other condition (such as retinal vessel obstruction or macular ischemia); and (5) history of retinal treatment, including laser, intravitreal injections, and other subtenon steroid injections, within 6 months before baseline evaluation.

All patients and controls had their best-corrected visual acuity (BCVA) measured initially and then underwent standardized dilated fundus examinations, including measurements by swept-source OCT and OCTA imaging (DRI OCT Triton; Topcon, Tokyo, Japan). In all patients, reevalu- ation after treatment was scheduled (as per usual clinical practice) 2 months after DEX implantation or 1 month after intravitreal anti-VEGF agent injection.

2.3. Treatment Protocol. The initial DEX implant $(0.7 \mathrm{mg})$ was injected into the vitreous cavity using standard protocols. After the first DEX implant injection, patients received a second treatment with anti-VEGF agents (Avastin ${ }^{\circledR}$; Genentech, Inc., San Francisco, CA) on an as-needed basis-pro re nata (PRN) dosing the regimen. The retreatment criterion was defined as central macular thickness $(\mathrm{CMT})>300 \mu \mathrm{m}$.

Patients were classified into two groups according to their treatment response: good-response group, comprising patients who received 4 or fewer treatments, and poorresponse group, comprising patients who received more than 5 treatments over 12 months from the initial treatment.

2.4. OCT Measurements. Swept-source OCT (DRI OCT Triton; Topcon) is a high-quality fundus imaging technique that relies on active eye tracking. The OCT images were generated using the horizontal OCT cross section (25 lines spaced $240 \mathrm{~mm}$ apart). CMT was measured using swept-source OCT (DRI OCT, Topcon, Japan). DME was defined as a $\mathrm{CMT}>300 \mu \mathrm{m}$.

2.5. OCTA Imaging and Analysis. OCTA was performed using the same device (DRI OCT Triton; Topcon). This device has an A-scan rate of 70,000 scans/s with an $840 \mathrm{~nm}$ wavelength light source and a $45 \mathrm{~nm}$ bandwidth. Patients with low-quality images (signal strength index $<50$ ) were excluded. OCTA images of the superficial capillary plexus (SCP), deep capillary plexus (DCP), and the choriocapillaris network were generated and segmented automatically by the built-in software (IMAGEnet 6, version 1.25). SCP was delineated by $2.6 \mu \mathrm{m}$ below the internal limiting membrane to $15.6 \mu \mathrm{m}$ below the junction, between the inner plexiform and the inner nuclear layers; DCP was delineated by $15.6 \mu \mathrm{m}$ below the inner plexiform and the inner nuclear layers to $70.2 \mu \mathrm{m}$ below them. Large intraretinal cysts in DME are often involved in multilayers and lead to inaccurate segmentation errors. For eyes with incorrect segmentation, we manually adjusted the offset value of the inner and outer borders for the DCP so that the DCP slab was segmented from just below the inner plexiform layer to just below the outer plexiform layer. Vessel density was defined as the percentage area occupied by vessels in a circular region centered on the center of the foveal avascular zone. Whole en face, foveal, and parafoveal vessel density of the SCP and DCP within 1 and $3 \mathrm{~mm}$ inner and outer circles was measured using computer software. We calculated the flow ratio as the ratio of the vessel density in the DCP to that in the SCP (flow ratio $=$ DCP vessel density/SCP vessel density) and determined the average values [12].

2.6. Statistical Analysis. Shapiro-Wilk test was used to assess the normality of data. A repeated measures ANOVA was used for the determination of the changes in visual acuity and in foveal thickness into the study patients. Unpaired $t$ -tests were used for between-group comparisons. Correlations between OCT parameters and the number of 
retreatments were assessed using Pearson's correlation test. Univariate and multivariate logistic regression models were used to identify the potential factors associated with retreatment. The receiver operating characteristic (ROC) curve and the area under the curve were used to assess the predictability of the DCP/SCP ratio for good treatment response in DME. $p$ values $<0.05$ were considered statistically significant. All analyses were performed using commercial software (SPSS version 22.0; IBM, Armonk, NY, USA).

\section{Results}

3.1. Patients' Characteristics. In total, 90 eyes of 90 patients (38 women and 52 men) with DME treated with DEX implant injection and 25 eyes of 25 age-matched control subjects were included. The baseline clinical and demographic characteristics of all study subjects are shown in Table 1.

3.2. Clinical and OCT Parameters. The mean BCVA significantly improved from $0.45 \pm 0.22 \operatorname{LogMAR}$ at baseline to $0.38 \pm 0.15$ LogMAR after 12 months of treatment $(p<0.001)$. Moreover, the mean CMT significantly reduced from $508.07 \pm 99.78 \mu \mathrm{m}$ at baseline to $277.04 \pm 65.23 \mu \mathrm{m}$ $(p<0.001)$ after 12 months of treatment. (Figure 1) The average time from intravitreal DEX injection to anti-VEGF was $4.33 \pm 1.13$ months (range 3-8). The total number of injections was $4.79 \pm 1.35$.

3.3. OCTA Parameters. Table 2 shows the SCP and DCP vessel density-whole, foveal, and parafoveal (superior/inferior/nasal/temporal)_in all patients. A statistically significant difference was observed between the DME and control groups in the microvascular parameters (Figure 2). In particular, the retinal vessel density in the whole and parafoveal areas of the DCP was significantly reduced in patients with DME compared with that in controls (all $p<0.05$ ). In the SCP, only the whole retinal vessel density was significantly reduced in patients with $\operatorname{DME}(p=0.026)$. The $\mathrm{DCP} / \mathrm{SCP}$ flow ratio was also significantly reduced in the DME group ( $1.08 \pm 0.03$ vs. $1.05 \pm 0.02, p=0.001)$.

3.4. Correlation Analysis. Among the OCTA parameters, the whole area vessel density in the DCP and the DCP/SCP flow ratio correlated significantly with the number of injections received $(p=0.015, r=-0.415$ and $p=0.025, r=-0.336$, respectively).

In the multivariate linear regression analyses for identifying factors related to the number of injections received, only the glycated hemoglobin level among the clinical parameters and the DCP/SCP flow ratio among the OCTA parameters showed a significant association $(\beta=-0.36, p=0.047$ and $\beta$ $=-1.003, p=0.017$, respectively).

3.5. Subgroup Analysis. There were 26 eyes in the goodresponse group and 64 eyes in the poor-response group. The foveal and whole vessel density in the DCP was significantly lower in the poor-response group than in the goodresponse group $(p=0.043$ and $p=0.048$, respectively) (Figure 1). Moreover, the DCP/SCP flow ratio was significantly lower in the poor-response group $(p=0.011)$
TABLE 1: Baseline characteristics of the diabetic macular edema study population.

\begin{tabular}{|c|c|}
\hline Characteristics & \\
\hline Age & $59.85 \pm 9.04$ \\
\hline $\operatorname{Sex}(M: F)$ & $52: 38$ \\
\hline DM duration (yr) & $13.46 \pm 7.32$ \\
\hline HbAlc & $7.14 \pm 0.66$ \\
\hline \multicolumn{2}{|l|}{ DR grade } \\
\hline Mild & 0 \\
\hline Moderate & 3 \\
\hline Severe NPDR & 48 \\
\hline PDR & 39 \\
\hline Hx of photocoagulation & 62 \\
\hline No. of treatment-naïve patients & $8(8.9 \%)$ \\
\hline BCVA (LogMAR) & $0.45 \pm 0.22$ \\
\hline Phakic eyes & $33(37 \%)$ \\
\hline Central macular thickness $(\mu \mathrm{m})$ & $508.07 \pm 99.78$ \\
\hline \multicolumn{2}{|c|}{$\begin{array}{l}\text { Data are presented as means } \pm \text { standard deviation, numbers, or numbers } \\
\text { (percentages). DM: diabetes mellitus; HbAlc: glycated hemoglobin; DR: } \\
\text { diabetic retinopathy; NPDR: nonproliferative diabetic retinopathy; PDR: } \\
\text { proliferative diabetic retinopathy; Hx: history; BCVA: best-corrected visual } \\
\text { acuity; LogMAR: logarithm of the minimum angle of resolution. }\end{array}$} \\
\hline
\end{tabular}

(Table 3). The ROC curve of the DCP/SCP flow ratio as a biomarker to predict poor treatment response is shown in Figure 3. The area under the curve was 0.702 . No significant differences were observed between the two groups in the SCP vessel density and the DCP parafoveal vessel density.

3.6. Side Effects. No injection-related complications were observed. Four eyes developed ocular hypertension and were treated with antihypertensive drops. Two of the phakic eyes (6\%) underwent cataract surgery due to the progression of lens opacity.

\section{Discussion}

In this study, we evaluated the OCTA microvascular parameters after a single intravitreal DEX implant injection followed by anti-VEGF therapy on a PRN basis and investigated their correlation with the number of injections received. DME was associated with significant retinal microvascular impairment in the DCP. There was a significant BCVA improvement and CMT reduction 12 months after the initial treatment. In patients with DME who had poor treatment response, there was a decreased DCP/SCP flow ratio.

We demonstrated that intravitreal DEX implant injection as initial therapy combined with anti-VEGF therapy on a PRN basis is effective for treating DME. In our study, we classified patients in the two response groups based on the number of treatments received. Busch et al. reported the outcomes of continued anti-VEGF therapy compared to switching DEX implant in eyes with DME in a real-world setting. At 


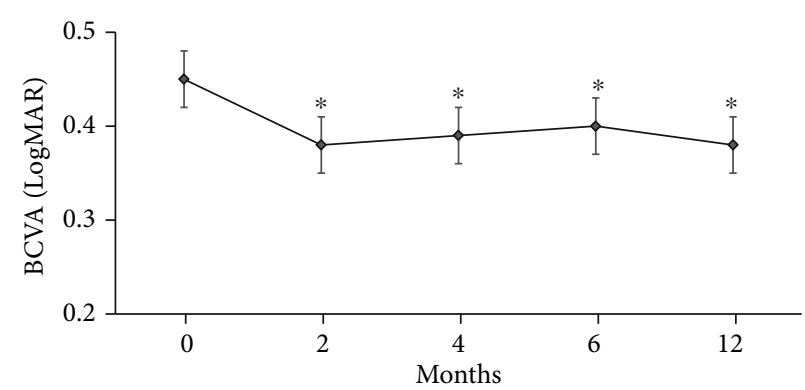

(a)

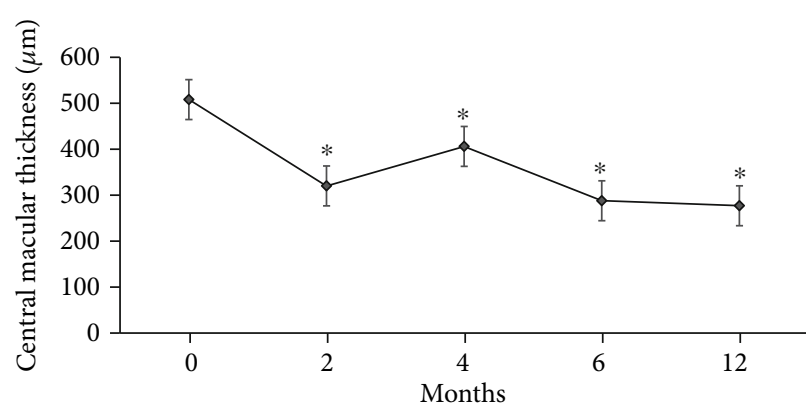

(b)

Figure 1: (a, b) Best-corrected visual acuity (BCVA) and central macular thickness (CMT) improved significantly compared to baseline during the observation period. ${ }^{*} p<0.001$; repeated measures ANOVA.

TABLE 2: Vessel density in patients with DME and controls.

\begin{tabular}{lccc}
\hline Characteristics & DME & Control & $p$ value \\
\hline $\begin{array}{l}\text { Superficial capillary plexus } \\
\text { Whole vessel density }\end{array}$ & $44.58 \pm 3.19$ & $46.04 \pm 2.29$ & $0.026^{*}$ \\
Foveal vessel density & $15.34 \pm 5.45$ & $15.23 \pm 3.31$ & 0.583 \\
Parafoveal vessel density & & & \\
$\quad$ Superior & $46.65 \pm 4.95$ & $48.7 \pm 4.54$ & 0.067 \\
$\quad$ Inferior & $45.72 \pm 5.27$ & $47.40 \pm 5.73$ & 0.167 \\
$\quad$ Temporal & $44.01 \pm 4.60$ & $45.74 \pm 3.31$ & 0.102 \\
$\quad$ Nasal & $41.94 \pm 5.27$ & $43.32 \pm 3.94$ & 0.243 \\
Deep capillary plexus & & & \\
Whole vessel density & $46.21 \pm 3.01$ & $49.55 \pm 3.01$ & $0.001^{*}$ \\
Foveal vessel density & $14.46 \pm 5.66$ & $14.50 \pm 2.74$ & 0.389 \\
Parafoveal vessel density & & & \\
$\quad$ Superior & $48.26 \pm 5.10$ & $52.51 \pm 4.64$ & $0.001^{*}$ \\
Inferior & $47.33 \pm 4.94$ & $50.86 \pm 5.78$ & $0.01^{*}$ \\
Temporal & $45.42 \pm 4.17$ & $48.46 \pm 3.81$ & $0.002^{*}$ \\
Nasal & $43.83 \pm 4.94$ & $46.38 \pm 4.15$ & $0.016^{*}$ \\
DCP/SCP ratio & $1.05 \pm 0.02$ & $1.08 \pm 0.03$ & $0.001^{*}$ \\
\hline Data are prested a mens & & &
\end{tabular}

Data are presented as means \pm standard deviation. DME: diabetic macular edema; DCP: deep capillary plexus; SCP: superficial capillary plexus.

12 months, mean anti-VEGF injections were $4.2 \pm 2.4$ except for the 3 loading injections. Therefore, we selected five treatments as the cutoff value $[13,14]$.

The pathophysiology of DME is complex and multifactorial; currently considered to be a chronic, low-grade inflammatory disorder [15]. It is associated with various vascular, neural, and glial cell components in the retina. Chronic hyperglycemia induces activation of the retinal glial cells that secrete VEGF and proinflammatory cytokines, leading to disruption in the blood-retinal barrier. Two widely used therapeutic strategies are the intravitreal steroid and intravitreal anti-VEGF injections [16-18].

Anti-VEGF agents, including ranibizumab, aflibercept, and bevacizumab, have been widely used for the treatment of DME for decades because of their convenience. However, the frequent injections due to the short duration of effect and the associated costs may be a heavy burden on the patients. In real-life settings, the treat-and-extend or PRN regimens have become popular as they decrease the treatment burden $[18,19]$.

DEX implants have demonstrated efficacy in the treatment of persistent DME, resistant to anti-VEGF treatment. Additionally, their effect is longer than that of anti-VEGF agents and six times stronger than that of triamcinolone acetonide $[20,21]$. Several studies have reported on the use of intravitreal DEX as initial therapy in patients with DME [22-24].

In the present study, we compared the degree of microvascular damage in relation to the treatment response and the DCP/SCP flow ratio was found to represent a response index. The vessel density in the whole area in the DCP and the DCP/SCP flow ratio correlated significantly with the number of injections. Furthermore, the DCP/SCP flow ratio was the only OCTA parameter significantly related to the number of injections in the multivariate linear regression analyses. Considering that the vessel density in OCTA demonstrates personal variations, the DCP/SCP flow ratio is remarkable. Yeung et al. reported that the DCP/SCP flow ratio in patients with branch retinal vein occlusion was associated with the treatment response $(p=0.015)$ and suggested that this ratio can represent the relative damage of the DCP to that of the SCP in BRVO [12]. We wondered that the hypothesis that the capillary loss in the DCP is more prominent than that in the SCP in DR may be relatively acceptable. Instead of absolute values in the individual layer of the capillary plexus, the ratio of the vessel density in the DCP to that in the SCP may be more meaningful.

In a previous study, Moon et al. reported that the DCP loss was more prominent in DME eyes than in non-DME eyes $[25,26]$. This trend was observed in anti-VEGF nonresponders compared with anti-VEGF responders. Altered vessel density in the DCP may imply a preference for this part of the retinal vascular system in the pathogenesis of DR or DME, as retinal venules originate from the deep retinal vascular layers. The earlier alterations in the deep vascular layer probably demonstrate retinal venular widening, damage to the capillary endings, and microaneurysms. It may also influence the breakdown of the blood-retinal barrier and the presence of DME [27-29]. 


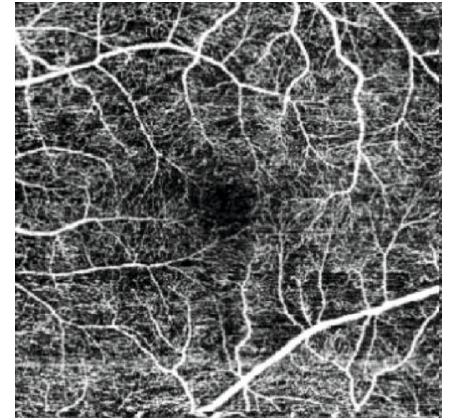

(a)

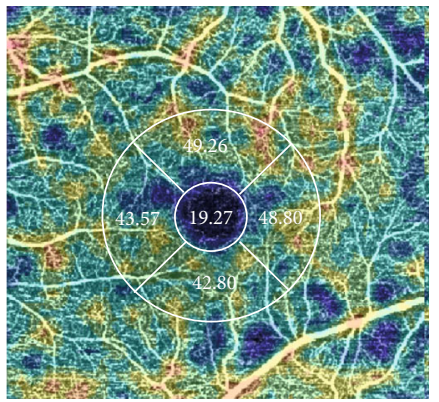

(c)

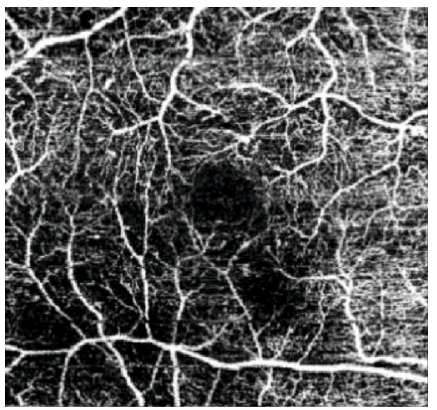

(e)

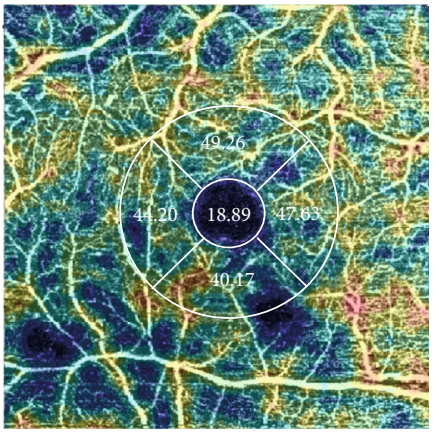

(g)

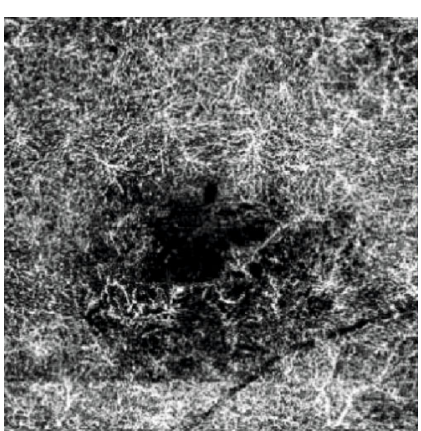

(b)

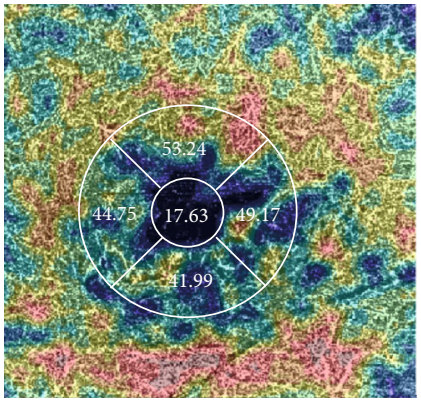

(d)

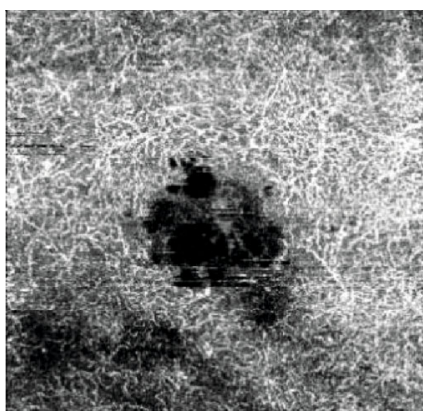

(f)

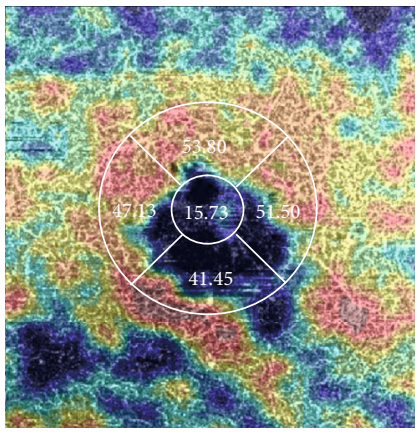

(h)

FIGURE 2: Representative samples of the microvascular parameters relative to the treatment response: (a-d) the parafoveal vessel density in the SCP and DCP (good-response group); (e-h) the parafoveal vessel density in the SCP and DCP (poor-response group).

In addition, we compared the two groups based on the number of treatment injections. The foveal and whole vessel density in the DCP and the DCP/SCP flow ratio were significantly lower in the poor-response group than in the good-response group. The underlying mechanism of the association between the low vessel density in the DCP and treatment inefficacy remains to be clearly defined. One possibility is excessive fluid flux from the vessels to the tissue because of the breakdown of the blood-retinal barrier caused by damage to the capillary endothelial tight junctions or various inflammatory cytokines $[30,31]$. The other suggestion is that the DCP might play a part in the removal of excess fluid from the retina. The smaller vessel density in the DCP could lead to fluid accumulation in the retina and would reduce fluid absorption $[32,33]$. 
TABLE 3: Clinical characteristics according to the DME treatment response.

\begin{tabular}{|c|c|c|c|}
\hline Characteristics & Good-response group & Poor-response group & $p$ value \\
\hline \multicolumn{4}{|c|}{ Superficial capillary plexus } \\
\hline Whole vessel density & $44.80 \pm 2.71$ & $44.49 \pm 3.34$ & 0.41 \\
\hline Foveal vessel density & $13.76 \pm 4.81$ & $15.98 \pm 5.52$ & 0.063 \\
\hline \multicolumn{4}{|l|}{ Parafoveal vessel density } \\
\hline Superior & $47.74 \pm 5.23$ & $46.2 \pm 4.73$ & 0.219 \\
\hline Inferior & $45.99 \pm 4.62$ & $45.61 \pm 5.47$ & 0.725 \\
\hline Temporal & $42.91 \pm 4.42$ & $44.46 \pm 4.56$ & 0.192 \\
\hline Nasal & $42.58 \pm 3.23$ & $41.67 \pm 5.13$ & 0.51 \\
\hline \multicolumn{4}{|l|}{ Deep capillary plexus } \\
\hline Whole vessel density & $47.13 \pm 2.12$ & $45.83 \pm 3.20$ & $0.043^{*}$ \\
\hline Foveal vessel density & $13.08 \pm 5.39$ & $15.03 \pm 5.62$ & $0.048^{*}$ \\
\hline \multicolumn{4}{|l|}{ Parafoveal vessel density } \\
\hline Superior & $49.5 \pm 4.95$ & $47.66 \pm 5.01$ & 0.094 \\
\hline Inferior & $48.44 \pm 4.41$ & $46.87 \pm 5.72$ & 0.31 \\
\hline Temporal & $44.89 \pm 3.85$ & $45.64 \pm 4.24$ & 0.504 \\
\hline Nasal & $45.45 \pm 3.35$ & $43.17 \pm 5.28$ & 0.081 \\
\hline $\mathrm{DCP} / \mathrm{SCP}$ ratio & $1.05 \pm 0.02$ & $1.02 \pm 0.03$ & $0.011^{*}$ \\
\hline Number of injections & $3.46 \pm 0.49$ & $5.47 \pm 0.9$ & $0.03^{*}$ \\
\hline
\end{tabular}

Data are presented as means \pm standard deviation. DME: diabetic macular edema; DCP: deep capillary plexus; SCP: superficial capillary plexus.

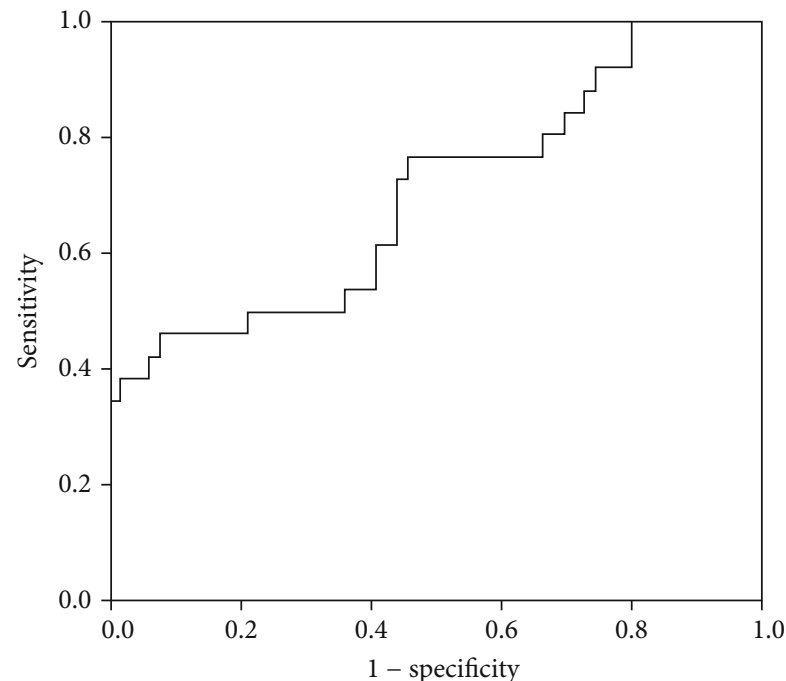

FIgure 3: The ROC curve of the DCP/SCP flow ratio for predicting the treatment response. The area under the ROC curve was 0.703 . ROC: receiver operating characteristics; DCP: deep capillary plexus; SCP: superficial capillary plexus.

To reduce ineffective repeated injections, it is important to screen for treatment-resistant DME in baseline examinations $[14,34]$. However, it is difficult to directly compare the result of various treatment regimens. Ebneter et al. reported a comparison between a PRN and a treat-andextend regimen in managing DME with intravitreal ranibizumab [35]. The mean number of injections was significantly different between the groups (PRN group: $5.9 \pm 1.8$; treat-and-extend group: $8.9 \pm 2.0 ; p<0.001)$. The VA of both groups showed similar gains $(8.3 \pm 6.7$ vs. $9.3 \pm 8.9$, $p=0.3$ ). In considering the duration of DEX implants, the number of retreatments in our study is not inferior to these results $(3.46 \pm 0.49$ vs. $5.47 \pm 0.9)$.

The most remarkable finding in our study was that the number of retreatments was related to the DCP/SCP flow ratio. Although several studies have evaluated the hard exudate or the microstructure with OCT to identify variables predicting the treatment response, we focused on the retinal microvascular changes evaluated with OCTA at baseline. In general, retinal vessel density was significantly reduced in patients with DME compared with that in controls. Compared with the SCP, vessel density rarefaction was prominent in the DCP, with the telangiectatic appearance of the retinal vessels. If the damage in the DCP is more severe than that in the SCP (in other words, considering the DCP/SCP ratio), the treatment response may be poor. Further studies are needed to identify predictors of the response to DME therapies and determine individualized therapeutic strategies according to the patients' features.

Our study has several limitations. First, the limited number of evaluated eyes led to low statistical power. Second, a selection bias may exist due to the retrospective study design. Third, most patients had a previous history of treatment and it may affect the outcomes. Fourth, the OCTA image artifacts can interfere with an accurate assessment of the actual status of the retinal microvasculatures. Projection artifacts might affect the visualization of the deep layer, and bias of segmentation error in cystoid macular edema may occur despite our best efforts to minimize such an effect. Last, it will be useful to observe the OCTA parameter changes, before and after the 
12-month treatment. However, it is difficult to consistently examine OCTA during follow-up periods in real-life practice. Regardless, this report provides a framework for further research.

\section{Conclusions}

The results of our study suggested that the OCTA retinal microvascular parameters at baseline influence the treatment outcomes in DME. Furthermore, decreased DCP/SCP flow ratio was observed in patients with DME who manifested poor treatment response. These parameters could represent predictors of the treatment response and would help to optimize the clinical outcomes.

\section{Data Availability}

The data used to support the findings of this study are available from the corresponding author upon request.

\section{Conflicts of Interest}

The authors have no conflict of interest with regard to this manuscript.

\section{Acknowledgments}

The authors wish to acknowledge the financial support of the Catholic Medical Center Research Foundation made in the program year of 2019 .

\section{References}

[1] N. Bhagat, R. A. Grigorian, A. Tutela, and M. A. Zarbin, "Diabetic macular edema: pathogenesis and treatment," Survey of Ophthalmology, vol. 54, no. 1, pp. 1-32, 2009.

[2] H. Funatsu, H. Noma, T. Mimura, S. Eguchi, and S. Hori, "Association of vitreous inflammatory factors with diabetic macular edema," Ophthalmology, vol. 116, no. 1, pp. 73-79, 2009.

[3] P. Brito, J. Costa, N. Gomes, S. Costa, J. Correia-Pinto, and R. Silva, "Serological inflammatory factors as biomarkers for anatomic response in diabetic macular edema treated with anti-VEGF," Journal of Diabetes and its Complications, vol. 32, no. 7, pp. 643-649, 2018.

[4] D. A. Antonetti, R. Klein, and T. W. Gardner, "Diabetic retinopathy," The New England Journal of Medicine, vol. 366, no. 13, pp. 1227-1239, 2012.

[5] L. Zhang, W. Wang, Y. Gao, J. Lan, and L. Xie, “The Efficacy and Safety of Current Treatments in Diabetic Macular Edema: A Systematic Review and Network Meta-Analysis," PLOS ONE, vol. 11, no. 7, p. e0159553, 2016.

[6] U. Schmidt-Erfurth, J. Garcia-Arumi, F. Bandello et al., "Guidelines for the management of diabetic macular edema by the European Society of Retina Specialists (EURETINA)," Ophthalmologica, vol. 237, no. 4, pp. 185-222, 2017.

[7] Y. He, X.-j. Ren, B.-j. Hu, W.-C. Lam, and X.-r. Li, "A metaanalysis of the effect of a dexamethasone intravitreal implant versus intravitreal anti-vascular endothelial growth factor treatment for diabetic macular edema," BMC Ophthalmology, vol. 18, no. 1, p. 121, 2018.
[8] G. Jian, X. Y. Jing, L. Yang, and L. Lun, "Quantitative Analysis of Foveal Microvascular Differences in Diabetic Macular Edema with and without Subfoveal Neuroretinal Detachment," Journal Diabetes Research, vol. 2020, article 2582690, pp. 1-7, 2020.

[9] S. Vujosevic, C. Toma, E. Villani et al., "Diabetic macular edema with neuroretinal detachment: OCT and OCTangiography biomarkers of treatment response to anti-VEGF and steroids," Acta Diabetologica, vol. 57, no. 3, pp. 287-296, 2020.

[10] F. J. Freiberg, M. Pfau, J. Wons, M. A. Wirth, M. D. Becker, and S. Michels, "Optical coherence tomography angiography of the foveal avascular zone in diabetic retinopathy," Graefe's Archive for Clinical and Experimental Ophthalmology, vol. 254, no. 6, pp. 1051-1058, 2016.

[11] L. Liu, Jian Gao, W. Bao et al., "Analysis of Foveal Microvascular Abnormalities in Diabetic Retinopathy Using Optical Coherence Tomography Angiography with Projection Artifact Removal," Journal of Ophthalmology, vol. 2018, Article ID 3926745, 9 pages, 2018.

[12] L. Yeung, W. C. Wu, L. H. Chuang, N. K. Wang, and C. C. Lai, "Novel optical coherence tomography angiography biomarker in branch retinal vein occlusion macular edema," Retina, vol. 39, no. 10, pp. 1906-1916, 2019.

[13] C. Busch, for the International Retina Group, S. Fraser-Bell et al., "Real-world outcomes of non-responding diabetic macular edema treated with continued anti-VEGF therapy versus early switch to dexamethasone implant: 2-year results," Acta Diabetologica, vol. 56, no. 12, pp. 13411350, 2019.

[14] C. Busch, For the International Retina Group, D. Zur et al., "Shall we stay, or shall we switch? Continued anti-VEGF therapy versus early switch to dexamethasone implant in refractory diabetic macular edema," Acta Diabetologica, vol. 55, no. 8, pp. 789-796, 2018.

[15] N. Cheung, P. Mitchell, and T. Y. Wong, "Diabetic retinopathy," Lancet, vol. 376, no. 9735, pp. 124-136, 2010.

[16] D. V. Do, Q. D. Nguyen, A. A. Khwaja et al., "Ranibizumab for Edema of the Macula in Diabetes Study," JAMA Ophthalmology, vol. 131, no. 2, pp. 139-145, 2013.

[17] U. Schmidt-Erfurth, G. E. Lang, F. G. Holz et al., "Three-year outcomes of individualized ranibizumab treatment in patients with diabetic macular edema: the RESTORE extension study," Ophthalmology, vol. 121, no. 5, pp. 1045-1053, 2014.

[18] C. Prünte, F. Fajnkuchen, S. Mahmood et al., "Ranibizumab $0.5 \mathrm{mg}$ treat-and-extend regimen for diabetic macular oedema: the RETAIN study," British Journal of Ophthalmology, vol. 100, no. 6, pp. 787-795, 2016.

[19] Y. J. Sepah, M. A. Sadiq, D. Boyer et al., "Twenty-four-month outcomes of the ranibizumab for edema of the macula in diabetes - protocol 3 with high dose (READ-3) study," Ophthalmology, vol. 123, no. 12, pp. 2581-2587, 2016.

[20] D. S. Boyer, Y. H. Yoon, Belfort R Jr et al., “Three-year, randomized, sham-controlled trial of dexamethasone intravitreal implant in patients with diabetic macular edema," Ophthalmology, vol. 121, no. 10, pp. 1904-1914, 2014.

[21] D. Zur, M. Iglicki, and A. Loewenstein, "The role of steroids in the management of diabetic macular edema," Ophthalmic Research, vol. 62, no. 4, pp. 231-236, 2019.

[22] S. K. Mahapatra and S. Kumari, "Long-term results of a single injection of intravitreal dexamethasone as initial therapy in 
diabetic macular edema," Indian Journal of Ophthalmology, vol. 68, no. 3, pp. 490-493, 2020.

[23] G. W. Blankenship, "Evaluation of a single intravitreal injection of dexamethasone phosphate in vitrectomy surgery for diabetic retinopathy complications," Graefe's Archive for Clinical and Experimental Ophthalmology, vol. 229, no. 1, pp. 6265, 1991.

[24] M. Iglicki, C. Busch, D. Zur et al., "Dexamethasone implant for diabetic macular edema in naive compared with refractory eyes," Retina, vol. 39, no. 1, pp. 44-51, 2019.

[25] S. A. Agemy, N. K. Scripsema, C. M. Shah et al., "Retinal vascular perfusion density mapping using optical coherence tomography angiography in normals and diabetic retinopathy patients," Retina, vol. 35, no. 11, pp. 2353-2363, 2015.

[26] B. G. Moon, T. Um, J. Lee, and Y. H. Yoon, "Correlation between deep capillary plexus perfusion and long-term photoreceptor recovery after diabetic macular edema treatment," Ophthalmol Retina, vol. 2, no. 3, pp. 235-243, 2018.

[27] K. Sambhav, K. K. Abu-Amero, and K. V. Chalam, "Deep capillary macular perfusion indices obtained with OCT angiography correlate with degree of nonproliferative diabetic retinopathy," European Journal of Ophthalmology, vol. 27, no. 6, pp. 716-729, 2017.

[28] R. F. Spaide, "Retinal vascular cystoid macular edema: review and new theory," Retina, vol. 36, no. 10, pp. 1823-1842, 2016.

[29] T. Mathis, T. Lereuil, A. Abukashabah et al., "Long-term follow-up of diabetic macular edema treated with dexamethasone implant: a real-life study," Acta Diabetologica, vol. 57, no. 12, pp. 1413-1421, 2020.

[30] J. K. Sun, M. M. Lin, J. Lammer et al., "Disorganization of the retinal inner layers as a predictor of visual acuity in eyes with center-involved diabetic macular edema," JAMA Ophthalmol, vol. 132, no. 11, pp. 1309-1316, 2014.

[31] A. Das, P. G. McGuire, and S. Rangasamy, "Diabetic macular edema: pathophysiology and novel therapeutic targets," Ophthalmology, vol. 122, no. 7, pp. 1375-1394, 2015.

[32] A. Couturier, V. Mané, S. Bonnin et al., "CAPILLARY PLEXUS ANOMALIES IN DIABETIC RETINOPATHY ON OPTICAL COHERENCE TOMOGRAPHY ANGIOGRAPHY," Retina, vol. 35, no. 11, pp. 2384-2391, 2015.

[33] R. Lazic, M. Lukic, I. Boras et al., "Treatment of anti-vascular endothelial growth factor-resistant diabetic macular edema with dexamethasone intravitreal implant," Retina, vol. 34, no. 4, pp. 719-724, 2014.

[34] G. Demir, A. Ozkaya, E. Yuksel et al., "Early and late switch from ranibizumab to an Intravitreal dexamethasone implant in patients with diabetic macular edema in the event of a poor anatomical response," Clinical Drug Investigation, vol. 40, no. 2, pp. 119-128, 2020.

[35] A. Ebneter, D. Waldmeier, D. C. Zysset-Burri, S. Wolf, and M. S. Zinkernagel, "Comparison of two individualized treatment regimens with ranibizumab for diabetic macular edema," Graefe's Archive for Clinical and Experimental Ophthalmology, vol. 255, no. 3, pp. 549-555, 2017. 